\title{
Gastrointestinal Non-Motor Dysfunction in Parkinson's Disease Model Rats with 6-hydroxydopamine
}

\author{
Xiao-Yan FENG ${ }^{1}$, Jing-Ting YAN ${ }^{1}$, Xiao-Li ZHANG ${ }^{1}$, Jin-Xia ZHU ${ }^{1}$ \\ ${ }^{1}$ Department of Physiology and Pathophysiology, School of Basic Medical Science, Capital \\ Medical University, Beijing, China
}

Received August 6, 2018

Accepted December 11, 2018

Epub Ahead of Print January 10, 2019

\begin{abstract}
Summary
Parkinson's disease (PD) is a neurodegenerative disease with a progressive loss of mesencephalic dopaminergic neurons of the substantia nigra (SN). To further evaluate its pathophysiology, accurate animal models are needed. The current study aims to verify the impact of a 6-hydroxydopamine (6-OHDA) bilateral microinjection into the $\mathrm{SN}$ on gastrointestinal symptoms in rats and confirm that the 6-OHDA rat model is an appropriate tool to investigate the mechanisms of Parkinsonian GI disorders. Immunohistochemistry, digital X-ray imaging, short-circuit current, FITC-dextran permeability and ultra-performance liquid chromatography tandem mass spectrometry were used in this study. The results indicated that the dopaminergic neurons in SN and fibres in the striatum were markedly reduced in 6-OHDA rats. The 6-OHDA rats manifested reductions in occupancy in a rotarod test and increases in daily food debris but no difference in body mass or daily consumption. Compared with control rats, faecal pellets and their contents were significantly decreased, whereas gastric emptying and intestinal transport were delayed in 6-OHDA rats. The increased in vivo FITC-dextran permeability and decreased intestinal transepithelial resistance in the model suggest attenuated barrier function in the digestive tract in the PD model. Moreover, inflammatory factors in the plasma showed that pro-inflammatory factors IL- $1 \beta$ and IL- 8 were significantly increased in 6-OHDA rats. Collectively, these findings indicate that the model is an interesting experimental tool to investigate the mechanisms involved in the progression of gastrointestinal dysfunction in PD.
\end{abstract}

\section{Key words}

Parkinson's disease - Gastrointestinal dysfunction • 6-hydroxydopamine

\section{Corresponding author}

Xiao-Yan Feng, Department of Physiology and Pathophysiology, School of Basic Medical Science, Capital Medical University, No. 10 Xitoutiao, You An Men, Beijing 100069, China. E-mail: fengxy@ccmu.edu.cn

\section{Introduction}

Parkinson's disease (PD) is a chronic, progressive dopaminergic neurological disorder, which is often accompanied by motor dysfunctions, such as resting tremor and rigidity, and various non-motor symptoms, especially gastrointestinal (GI) dysfunctions including gastroparesis, constipation and duodenal ulcer (Odin et al. 2018, Sauerbier et al. 2017, Shen et al. 2017). It has been reported that motor symptoms are realized after a loss of more than $70 \%$ of the dopaminergic neurons in the substantia nigra (SN) (Ferro et al. 2005), but a modest reduction in dopamine content is sufficient to cause GI dysfunction before the occurrence of motor disorders (Zheng et al. 2014). Clinical research also suggests that GI dysfunctions frequently appear in the early stages of the disease or even many years before motor impairment.

Animal models have been acknowledged as useful and important tools to analyse the pathogenic mechanisms of manifestations and potential therapeutic agents in PD (Grandi et al. 2018, Jakaria et al. 2018). Neurotoxic agents include rotenone, 6-hydroxydopamin (6-OHDA), lipopolysaccharide (LPS) and 1-methyl-4phenyl-1,2,3,6-tetrahydropyridine (MPTP) (Johnson et al. 2015, Marin et al. 2015) and dopaminergic neurotransmission drugs, such as reserpine (Shireen et al. 
2014) or genetic manipulation (Imbriani et al. 2018), have been administered to animals to mimic the characteristic symptoms of PD. In all of the above cases, unilateral administration of 6-OHDA to the medial forebrain bundle (MFB) (Boix et al. 2015) or the SN (Kim et al. 2016) was the most widely used PD models. However, PD affects both brain hemispheres, and GI functions are bilaterally controlled. Compared to the unilateral 6-OHDA rat model, bilateral models more closely approximate the real pathological situation and exclude compensation for the lesion side by the intact site (Deumens et al. 2002). In the present study, we aimed to verify the impact of bilateral microinjection of 6-OHDA into the $\mathrm{SN}$ on gastrointestinal symptoms in rats and confirm the 6-OHDA rat is an appropriate experimental tool to investigate the mechanisms involved in the progression of GI dysfunction in PD. Metabolic measurement, digital X-ray imaging, short-circuit current and FITC-dextran permeability were used to determine the reproducibility of the bilateral 6-OHDA model and its ability to the mimic the many physiological features of PD.

\section{Methods}

\section{Drugs and solutions}

Dopamine hydrochloride, 6-hydroxydopamine hydrochloride, FITC-dextran (Sigma-Aldrich, St. Louis, MO, USA), and barium meal (Kangte Biological Engineering Co., Ltd, Jiangsu, China) were used in the present study. The Krebs-Henseleit solution (K-HS) contained the following (in mmol/l): $\mathrm{NaCl} \mathrm{117,} \mathrm{KCl} 4.7$, $\mathrm{MgCl}_{2} \cdot 6 \mathrm{H}_{2} \mathrm{O}$ 1.2, $\mathrm{CaCl}_{2} \cdot 2 \mathrm{H}_{2} \mathrm{O} \quad 2.5, \mathrm{NaHCO}_{3} \quad 24.8$, $\mathrm{KH}_{2} \mathrm{PO}_{4} 1.2$ and glucose 11.1. The solution was gassed with $95 \% \mathrm{O}_{2}$ and $5 \% \mathrm{CO}_{2}$, and $\mathrm{HCl}$ was used to adjust the $\mathrm{pH}$ to 7.4 .

\section{Animals and tissue preparation} Animal Care

All male Sprague-Dawley rats (210-230 g) were purchased and maintained in the animal facilities at the Laboratory Animal Services Center of Capital Medical University. The animals were housed in a light-dark cycle of 12:12 $\mathrm{h}$ and provided free access to food and water. All of the experiments were performed in accordance with the guidelines established by the Beijing Administration Office of Laboratory Animals and following the Administration Regulations on Laboratory Animals of Beijing Municipality.

\section{6-OHDA Rats}

The methods for producing 6-OHDA rats have been previously described (Feng et al. 2017). Briefly, the rats were received bilateral infusions of 6-OHDA ( $4 \mu \mathrm{g}$ in $2 \mu \mathrm{l}$ of $0.9 \%$ saline containing $0.05 \%$ ascorbic acid for each injection site) into the SN using a $10 \mu$ l Hamilton syringe. The control rats received $0.2 \%$ ascorbic acid/saline.

At 4 weeks after 6-OHDA treatment, each rat was transferred into an individual metabolic cage (Ugo Basile, Gemonio VA, Italy) and observed throughout a $24 \mathrm{~h}$ period to monitor the daily food and water consumption for one week. The food residue and stool samples were collected and measured every day during the fifth week. The solid matter of the stool was dried in an oven at $60{ }^{\circ} \mathrm{C}$ for $12 \mathrm{~h}$.

\section{Tissue preparation}

The rats were killed by decapitation at the sixth week. The brains were immediately removed and immersed in $4 \%$ paraformaldehyde $(12 \mathrm{~h})$ for postfixation and then placed in $30 \%$ sucrose $(48 \mathrm{~h})$ for dehydration. The brains were retained for immunohistochemistry. Then, the abdominal wall was opened. The duodenum next to the gastric antrum and the distal colonic segment away from the anus (approximately $2 \mathrm{~cm}$ ) was quickly removed and immersed in K-HS. Each segment $(1 \mathrm{~cm})$ was cut longitudinally along the mesenteric border and cleaned. The duodenal/colonic tissue was pinned (mucosal side down) in a Sylgard-lined Petri dish to strip away the serosa, muscularis and submucosa with fine forceps. The duodenal/colonic mucosa preparations were obtained for in vitro short circuit current measurement.

\section{Immunohistochemistry}

The brain slices were fixed with cold acetone for $15 \mathrm{~min}$, and then washed $(3 \times 5 \mathrm{~min})$ in $0.3 \%$ Triton $\mathrm{X}-100$ phosphate buffer solution (PBST) to eliminate the residual fixative. After blocking with $3 \% \mathrm{H}_{2} \mathrm{O}_{2}$ and $10 \%$ goat serum (Sigma-Aldrich, St. Louis, MO, USA) at room temperature for $30 \mathrm{~min}$, the sections were incubated with TH antibody (Mouse, 1:10000, Sigma/T1299) at $4{ }^{\circ} \mathrm{C}$ overnight. After washing in PBST $(3 \times 5 \mathrm{~min})$, sections of the $\mathrm{SN}$ were incubated with donkey antimouse IgG (1:1000, Invitrogen/A21203) for $1 \mathrm{~h}$ at room temperature and then observed under a fluorescence microscope (Leica DM LB2, St. Gallen, Switzerland). The sections of the striatum were incubated with 
sheep anti-mouse IgG (1:1000, Rockland/13175) for $1 \mathrm{~h}$ at room temperature and incubated with 3, 3'-diaminobenzidine tetrahydrochloride (DAB Substrate Kit for Peroxidase, Beyotime Biotechnology, Shanghai, China) for $2 \mathrm{~min}$, stopped with water, and then placed in xylene and overlaid with a coverslip using neutral resin-mounting medium.

\section{Ultra-performance liquid chromatography tandem mass spectrometry (UPLC-MS/MS)}

The SN, striatum and DMV tissues were harvested from the rats at the sixth week. The DA content in these tissues was measured by UPLC-MS/MS analysis, which has been described elsewhere (Zhang et al. 2015). Briefly, each sample was weighed and homogenized in $2 \%$ aqueous formic acid. The homogenates were ultrasonically dissociated with a mixture of acetonitrile/methanol/formic acid and centrifuged. The supernatant was evaporated to dryness and re-dissolved with reconstitution solvent followed by another round of centrifugation. The supernatant was immediately used for UPLC-MS/MS analysis (Key Laboratory of Radiopharmaceuticals, Ministry of Education, College of Chemistry, Beijing Normal University).

\section{Rotarod test}

A rotarod test was used to evaluate motor coordination by measuring the ability of a rat to stay on a rotating drum. At the fifth week after 6-OHDA treatment, the rats were placed on the rotarod instrument (diameter 3.75 inches, Acceler Rotarod, Jones \& Roberts Company, Olympia) at a fixed speed for adaptive training before testing (3-5 min, 3 times each day for 3 consecutive days). On the test day, the rats were trained for 3-5 $\mathrm{min}$ until they adapted to $8 \mathrm{rpm} / \mathrm{min}$ on the rotarod. After the rats were balanced, the drum was gradually accelerated until the rats fell off of the drum. The time and speed to fall was recorded by a sensing platform. Each rat was given 3 trials, and the mean time of the 3 trials was calculated.

\section{Gastrointestinal motility}

Gastric emptying was assessed using an in vivo digital X-ray imaging. Each animal received $3 \mathrm{ml}$ of a barium meal (barium sulphate) through oral gavage after fasting for $20 \mathrm{~h}$. The Kodak In Vivo Imaging System FX was used to obtain the gut plain radiographs with a manual focus distance of $50 \pm 1 \mathrm{~cm}$ and an exposure time of $30 \mathrm{~s}$. The images were recorded every $45 \mathrm{~min}$ after barium meal ingestion. The barium sulphate content in the stomach was measured with area and greyscale. The intestinal motility was measured by the total intestinal transit time, which was recorded by the first stool including barium sulphate.

\section{Short-circuit current (ISC) measurement}

The duodenal/colonic mucosa preparations were mounted between the two halves of an Ussing chamber, bathed in $5 \mathrm{ml} \mathrm{K}$-HS $\left(37{ }^{\circ} \mathrm{C}\right)$ in both sides and gassed with $95 \% \mathrm{O}_{2}$ and $5 \% \mathrm{CO}_{2}$. The transepithelial potential difference for each preparation was measured with the Ussing chamber system (Physiologic Instruments, San Diego, CA, USA, VCC MC6).

\section{In vivo permeability measurement}

The assay was slightly modified from the previously described methods (Moussaoui et al. 2016). Briefly, the rats were given an oral gavage of fluorescein isothiocyanate (FITC)-dextran (4.4kD) at a final dose of $600 \mathrm{mg} / \mathrm{kg}$ in PBS at 9:00 am. After $4 \mathrm{~h}$, a blood sample was taken from each rat by cardiac puncture. The blood was centrifuged at $4{ }^{\circ} \mathrm{C}, 3000 \times \mathrm{g}$ for $20 \mathrm{~min}$, and the plasma was taken for the analysis of the FITC-dextran concentration. The plasma was diluted at 1:2 with PBS, and the fluorescence intensity of the diluted plasma was then measured by using a fluoro-spectro photometer (Hitachi Ltd, Tokyo, Japan) with an excitation wavelength of $480 \mathrm{~nm}$ and an emission wavelength of $520 \mathrm{~nm}$. The plasma FITC-dextran concentrations were calculated from standard curves generated by serial dilution of FITC-dextran in control plasma.

\section{Statistical analysis}

The results are given as arithmetic means \pm SEM, " $n$ " refers to the number of rats or the number of pairs. Statistical analyses included the Student's paired or unpaired $t$-test. Statistics and graphs were generated by using GraphPad Prism, version 5.0 (GraphPad Software, San Diego, Calif., USA). " $p$ " values less than 0.05 were assumed to denote a significant difference.

\section{Results}

\section{Characterization of the bilateral 6-OHDA lesions}

Tyrosine hydroxylase (TH), which is the ratelimiting enzyme of DA synthesis, acts as the most important dopaminergic marker. The results indicated that the TH-immunoreactive dopaminergic neurons in SN 
A
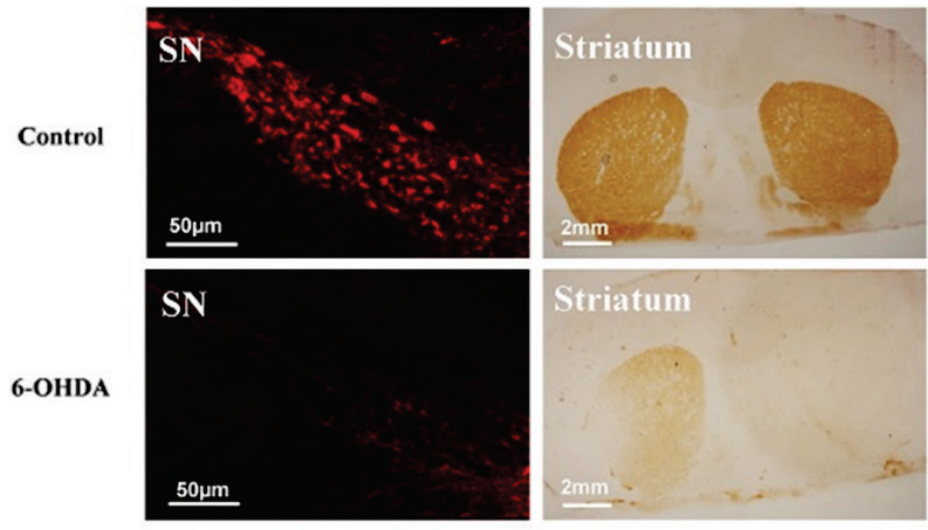

Striatum

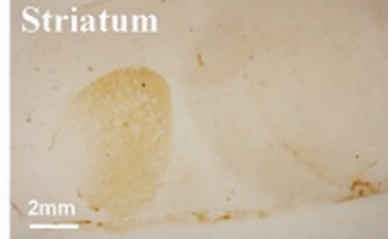

B

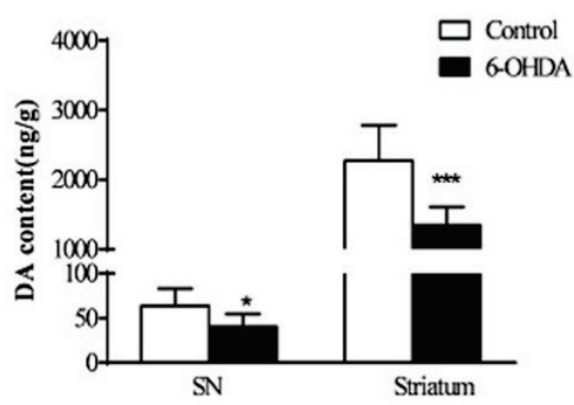

$\mathbf{E}$

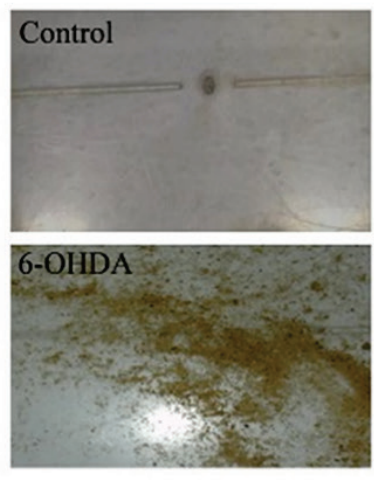

Fig. 1. Characterization of bilateral 6-OHDA lesions. (A, B) The TH immunoreactivity and DA content in the SNs and striata of control and 6-OHDA rats. (C) The rotarod test results of control and 6-OHDA rats. (D) The original recording showing daily food debris of control and 6-OHDA rats. (E) A summary of the daily food debris of the control and 6-OHDA rats. Values are the means \pm S.E.M. $* p<0.05, * * p<0.01$, and $* * * p<0.001$

and the fibres in the striatum were significantly reduced in 6-OHDA rats (Fig. 1A). Compared to the control rats, the DA contents in the $\mathrm{SN}$ and striatum were markably decreased from $63.7 \pm 6.9 \mathrm{ng} / \mathrm{g}$ to $40.4 \pm 4.9 \mathrm{ng} / \mathrm{g}(n=8$, $p<0.05)$ and from $2270.0 \pm 181.2 \mathrm{ng} / \mathrm{g}$ to $1344.0 \pm 95.9 \mathrm{ng} / \mathrm{g}$ $(n=8, p<0.001)$, respectively (Fig. 1B).

At the fifth week after 6-OHDA treatment, each rat was transferred into an individual metabolic cage to its monitor body mass and daily consumption for one week. No difference in body mass $(399.7 \pm 5.6 \mathrm{~g}$ vs. $389.7 \pm 6.2 \mathrm{~g})$, food $(30.2 \pm 1.3 \mathrm{~g}$ vs. $29.1 \pm 2.9 \mathrm{~g})$ and water consumption $(27.4 \pm 1.2 \mathrm{~g}$ vs. $26.6 \pm 1.3 \mathrm{~g})$ were observed between the control and the 6-OHDA rats $(n=12$, $p>0.05$ ). However, the rotarod test results showed decreased treadmill occupancy times in 6-OHDA rats $(n=12, p<0.001)$ (Fig. 1C), which suggests that the lesion of dopaminergic neurons in the $\mathrm{SN}$ caused motor coordination and balance function disorder. As shown in Fig. 1D, the food residue was detected at the bottom of the metabolic cage and collected throughout a $24 \mathrm{~h}$ period for measurement. The increased daily food debris of 6-OHDA rats, from $0.3 \pm 0.1 \mathrm{ng} / \mathrm{g}$ to $3.1 \pm 0.2 \mathrm{ng} / \mathrm{g}(n=12$, $p<0.001)$, suggested the emergence of rigidity in the PD model ( $n=10, p<0.001)$ (Fig. 1E).

\section{Gastrointestinal motility dysfunction of the 6-OHDA rats}

An in vivo digital $\mathrm{X}$-ray imaging system was used to evaluate gastric emptying and intestinal transit time. Following a $20 \mathrm{~h}$ fast, $3 \mathrm{ml}$ of a barium sulphate suspension was administered to each rat at room temperature. Images were recorded every $45 \mathrm{~min}$ after barium meal ingestion. The results indicated that the gastric areas of 6-OHDA rats were significantly larger than those of the control group, but gastric emptying and intestinal barium meal transit were apparently slower in all images throughout the experiment (Fig. 2A). After barium meal intragastric administration for $3 \mathrm{~h}$, the gastric areas were obviously increased from $0.69 \pm 0.06$ in the control group to $0.96 \pm 0.06$ in the 6-OHDA group ( $n=7, p<0.01)$; however, compared to the control stomach content emptying of $74.11 \pm 4.37 \%$, only $37.30 \pm 2.90 \%$ of the stomach contents were emptied in the 6-OHDA rats $(n=7, p<0.001)$ (Fig. $2 \mathrm{~B})$. Intestinal transit is most often measured as the total intestinal transit time along 
the entire alimentary tract and is mainly a function of propulsion in the small and large intestine. To confirm the intestinal transit time, the time of the first stool including barium meal was recorded. The 6-OHDA rats produced a longer transit time after barium meal intragastric administration from $339 \pm 14 \mathrm{~min}$ to
$483 \pm 26 \min (n=8, p<0.001)$ (Fig. $2 \mathrm{C})$. Furthermore, the number of daily faecal pellets and the faecal content, including solid matter $(n=15, p<0.001)$ and moisture $(n=15, p<0.05)$, were significantly decreased in 6-OHDA rats (Fig.2 D-F), which indicated the impairment of GI motility.
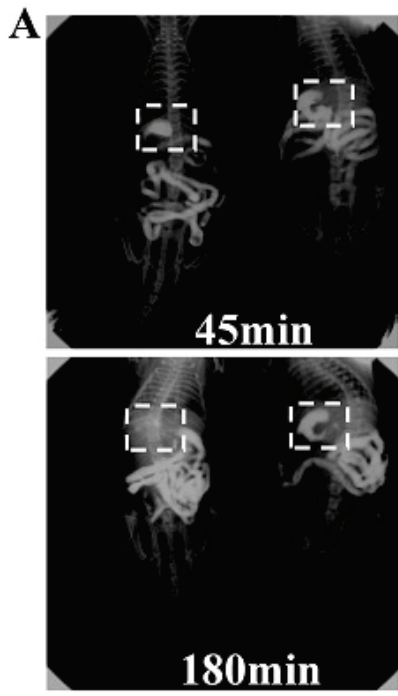

D
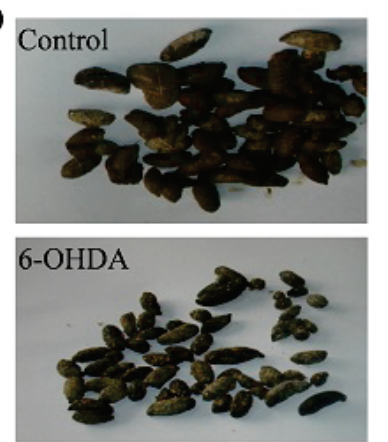
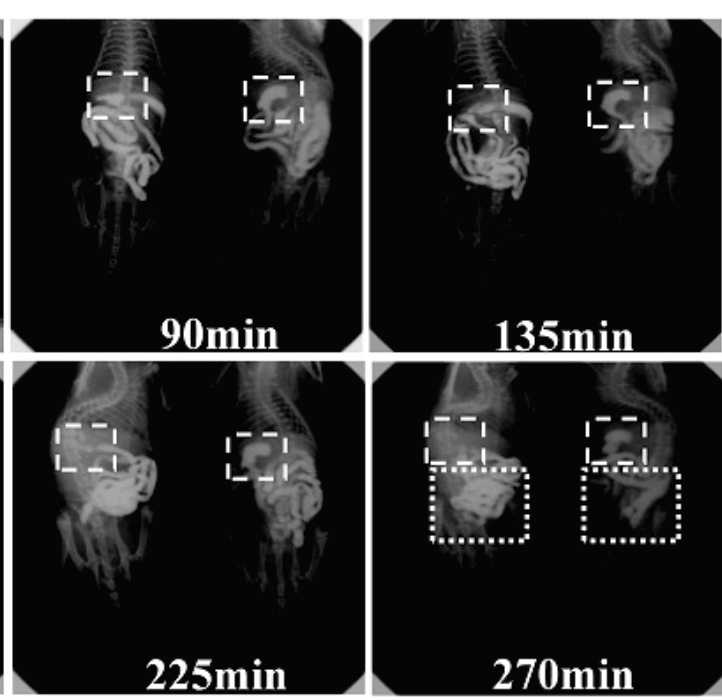

$\mathbf{E}$
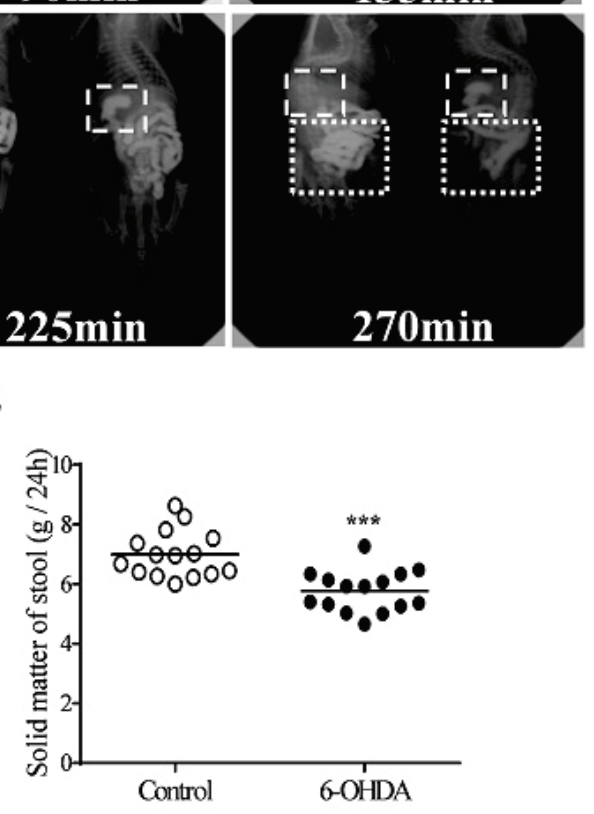

B
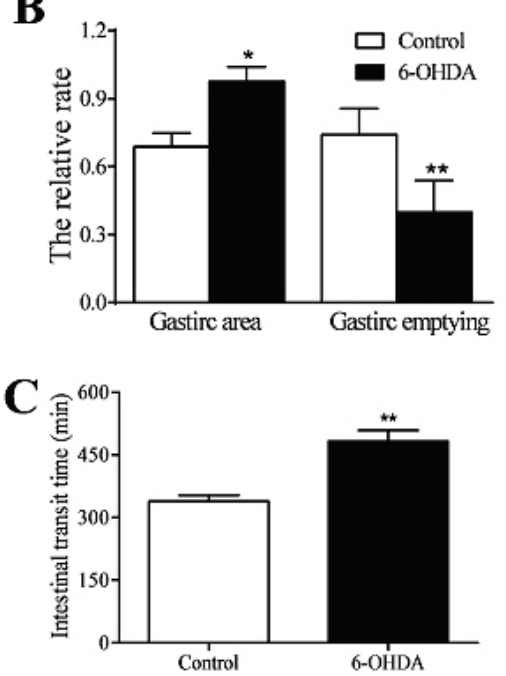

$\mathbf{F}$

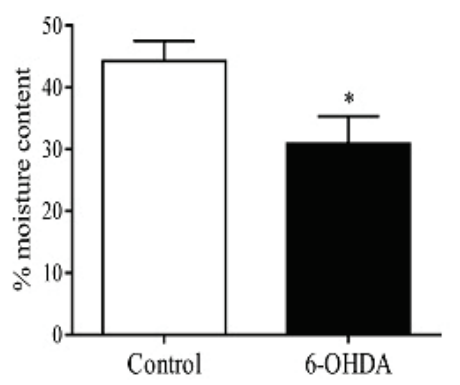

Fig. 2. Gastrointestinal motility dysfunction of the 6-OHDA rats. (A) The original images of gastric emptying every 45 min after barium meal ingestion in control and 6-OHDA rats. (B) The gastric area and emptying of the barium meal after $3 \mathrm{~h}$ in control and 6-OHDA rats. (C) The total intestinal transit time in control and 6-OHDA rats. (D-F) The number of stools and solid matter and moisture content of faeces in control and 6-OHDA rats. Values are means \pm S.E.M. ${ }^{*} p<0.05, * * p<0.01$, and ${ }^{*} * * p<0.001$.

Gastrointestinal barrier dysfunction of the 6-OHDA rats

The FITC-dextran concentration was determined from analysis of the standard curve of dextran-FITC using a 96-well microplate fluorescence reader. Compared with the control rats, the 6-OHDA rats showed increased FITC-dextran permeability from $0.16 \pm 0.02 \mu \mathrm{g} / \mathrm{ml}$ to $0.23 \pm 0.02 \mu \mathrm{g} / \mathrm{ml}$ by in vivo measurement ( $n=9, p<0.01)$ (Fig. 3A), whereas intestinal transepithelial resistance (TER) decreased from $46.36 \pm 2.82 \Omega / \mathrm{cm}^{2}$ to $35.38 \pm 3.52 \Omega / \mathrm{cm}^{2}(n=12, p<0.05)$ in the duodenal preparations and from $95.19 \pm 4.88 \Omega / \mathrm{cm}^{2}$ to $62.60 \pm 4.89 \Omega / \mathrm{cm}^{2} \quad(n=12, p<0.05)$ in the colonic preparations (Fig. 3B), which suggested that the intestinal mucosal barrier was impaired. A link between alterations in inflammatory factors and GI dysfunction, especially intestinal permeability, has been reported (Netusha et al. 2008). Therefore, inflammatory factors in the plasma were detected. The results showed that the pro-inflammatory factors IL- $1 \beta(n=7, p<0.05)$ and IL- 8 $(n=7, \quad p<0.01)$ were significantly increased, and the anti-inflammatory factor IL-10 $(n=7, p<0.01)$ was decreased in 6-OHDA rats. 
A

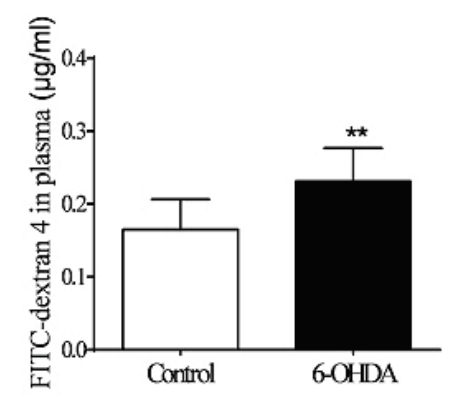

B

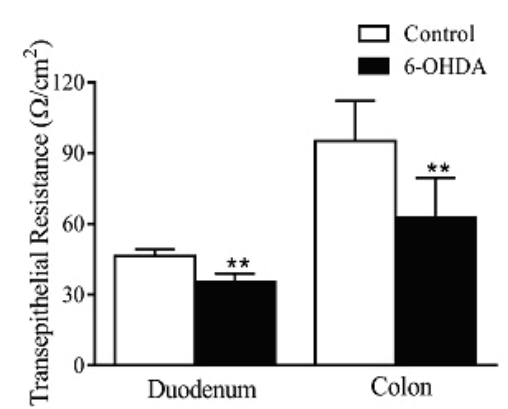

C

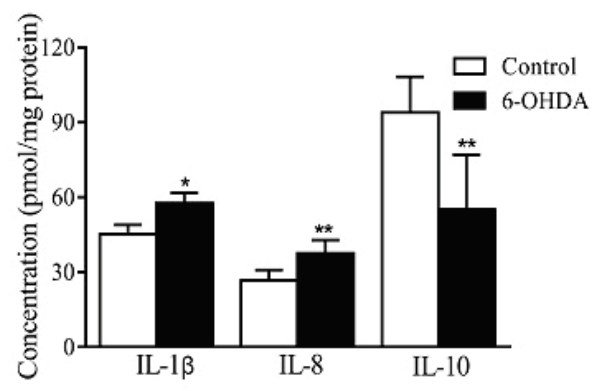

Fig. 3. Gastrointestinal barrier dysfunction of the 6-OHDA rats. (A) The FITC-dextran concentration in the plasma of control and $6-O H D A$ rats. (B) The TERs of duodenal and colonic preparations in the control and 6-OHDA rats. (C) The inflammatory factors in the plasma of control and 6-OHDA rats. Values are means \pm S.E.M. $* p<0.05, * * p<0.01$, and $* * * p<0.001$.

\section{Discussion}

Although the 6-OHDA rats employed in the present study did not mimic all clinical and pathological symptoms of PD patients, the data in the present study provided evidence that this animal model is a useful tool to investigate the mechanisms of Parkinsonian GI disorders. GI dysfunction, together with sleep dysfunction, dysosmia, and other dysautonomia are included in the non-motor symptoms (NMS) of PD, which are key components of $\mathrm{PD}$ and present from the 'pre-motor' phase to the final palliative stage (Zis et al. 2015). Therefore, using the bilateral 6-OHDA model to study the mechanisms involved in the progression of GI dysfunction in PD would benefit the quality of life in PD patients.

The main neuropathological features of PD are the loss of dopaminergic neurons in the $\mathrm{SN}$ and their projections into the caudate nucleus (Zheng et al. 2011). The results from immunohistochemistry and UPLC-MS/MS showed significant depression of the TH-immunoreactive signalling and DA content of the SN and striatum of the 6-OHDA rats. It has been reported that a direct or indirect connection may exist between the SN-striatum and the DMV (Zheng et al. 2011), and DA modulates the neurons in the DMV, which may contribute the impaired gastric motility (Anselmi et al. 2017). Furthermore, activating $D_{1} R$ or $D_{2} R$ in the DMV can hyperpolarize or depolarize the membrane potential of DMV neurons innervating the GI tract, especially those in the stomach (Zheng et al. 2007).

GI dysfunction is often considered an essential PD symptom that dominates the clinical outlook for some patients and is mostly represented by gastroparesis, constipation and peptic ulcers (Jiang P et al. 2018, Fornai et al. 2016). It has been found that 6-OHDA rats had enhanced expression of dopaminergic markers, which suggests a significant increase of DA content in the guts of 6-OHDA rats (Tian et al. 2008). However, increased DA and reduced acetylcholine content in the gastric muscularis externa lead to impaired gastric motility in 6-OHDA rats (Zheng et al. 2014). Zhang et al. also reported that high DA levels and upregulated $\mathrm{D}_{1}$ receptors in smooth muscle resulted in an enhanced inhibitory effect on colonic contraction in the cold-restraint stress condition (Zhang et al. 2012). The radiological findings regarding delayed intestinal transit have been previously observed in 6-OHDA unilateral administration rats, which is also due to an impairment of acetylcholine release from colonic myenteric neurons (Fornai et al. 2016). In the present study, we applied an in vivo digital X-ray imaging system to confirm impaired gastric emptying and intestinal transit time in 6-OHDA rats. The alterations of the enteric neurotransmitters (DA, acetylcholine, nitric oxide, and vasoactive intestinal peptide) involved in the regulation of intestinal motility in the 6-OHDA model suggest that central dopaminergic neurodegeneration is associated with remodelling of enteric neurotransmission (Pellegrini et al. 2016). In contrast, the intraperitoneal MPTP-lesioned mice showed a loss of dopaminergic neurons both in the SN and in the gastric wall (Tian et al. 2008, Natale et al. 2010), while the MPTP-based animal models showed no significant changes in gastric emptying or intestinal transit time (Anderson et al. 2007).

Mucosal barrier damage with high permeability and bowel inflammation plays an important role in peptic ulcer formation (Feng et al. 2017). Intestinal permeability can be assessed in vivo by determining the permeability of FITC-dextran with a defined molecular size in the blood plasma. TER is another common physiological index used to evaluate the mucosal barrier. Our study 
showed that 6-OHDA rats had increased FITC-dextran permeability and decreased TER in the gut, thus indicating attenuated mucosal integrity (Tulstrup et al. 2015). Moreover, the GI barrier can provide an immune sentinel function by secreting various cytokines in the bacterial stimulation, including the IL-1 family (e.g., IL-1 $\beta$, IL-18, and IL-33), IL-6, IL-8, and some antiinflammatory cytokines (e.g., IL-10 and IL-25). Studies have shown that IL-1 $\beta$ participates in the inflammatory responses by augmenting the infiltration of neutrophils via the activation $\mathrm{T}$ cells and innate lymphoid cells (Sun et al. 2017). Our results showed that 6-OHDA rats displayed a chronic intestinal disorder, which was caused by an exaggerated immune response with increased pro-inflammatory factors IL- $1 \beta$ and IL- 8 and decreased anti-inflammatory factor IL-10. Interestingly, similar results have also been reported by Pellegrini et al. in that there were increased levels of MDA, TNF, and IL-1 $\beta$ in colonic walls isolated from 6-OHDA rats, which suggests the presence of gut inflammation and oxidative stress in the colonic wall (Pellegrini et al. 2016).

In conclusion, our findings suggest that 6-OHDA rats would be an available PD model to investigate the mechanisms involved in the progression of GI non-motor dysfunctions and improve the quality of life of PD patients though drug treatment and more effective assistance.

\section{Acknowledgements}

This work was financially supported through grants from National Key Research and Development Program (2016YFC1302203, Zhu JX) and National Natural Science Foundation of China (31500937, Feng XY).

\section{Conflict of Interest}

All the authors of the manuscript have read the journal's policy on disclosure of potential conflicts of interest and agreed to its content. The manuscript is original, has not already been published in any other journal and is not currently under consideration by any another journal.

\section{Abbreviations}

PD, Parkinson's disease; DA, dopamine; GI, gastrointestinal; 6-OHDA, 6-hydroxydopamine; K-HS, Krebs-Hensleit solution; $I_{S C}$, short-circuit current; UPLC$\mathrm{MS} / \mathrm{MS}$, ultra-performance liquid chromato-graphy tandem mass spectrometry; PBST, phosphate buffer solution; TER, transepithelial resistance;

\section{References}

ANDRSON G, NOORIAN AR, TAYLOR G, ANITHA M, BERNHARD D, SRINIVASAN S, GREENE JG: Loss of enteric dopaminergic neurons and associated changes in colon motility in an MPTP mouse model of Parkinson's disease. Exp Neurol 207: 4-12, 2007.

ANSELMI L, TOTI L, BOVE C, TRAVAGLI RA: Vagally mediated effects of brain stem dopamine on gastric tone and phasic contractions of the rat. Am J Physiol Gastrointest Liver Physiol 313: 434-441, 2017.

BOIX J, PADEL T, PAUL G: A partial lesion model of Parkinson's disease in mice-characterization of a 6-OHDAinduced medial forebrain bundle lesion. Behav Brain Res 284: 196-206, 2015.

DEUMENS R, A BLOKLAND, PRICKAERTS J: Modeling Parkinson's disease in rats: an evaluation of 6-OHDA lesions of the nigrostriatal pathway. Exp Neurol 175: 303-317, 2002.

FENG XY, LI Y, LI LS, LI XF, ZHENG LF, ZHANG XL, FAN RF, SONG J, HONG F, ZHANG Y, ZHU JX: Dopamine D1 receptors mediate dopamine-induced duodenal epithelial ion transport in rats. Transl Res 161: 486-494, 2013.

FENG XY, ZHANG DN, WANG YA, FAN RF, HONG F, ZHANG Y, LI Y, ZHU JX: Dopamine enhances duodenal epithelial permeability via the dopamine $\mathrm{D}_{5}$ receptor in rodent. ACTA Physiologia (Oxf). 220: 113-123, 2017.

FERRO MM, BELLISSIMO MI, ANSELMO-FRANCI JA, ANGELLUCCI ME, CANTERAS NS, DA CC: Comparison of bilaterally 6-OHDA- and MPTP-lesioned rats as models of the early phase of Parkinson's disease: histological, neurochemical, motor and memory alterations. J Neurosci Methods 148: 78-87, 2005.

GRANDI LC, Di GIOVANNI G, GALATI S: Animal models of early-stage Parkinson's disease and acute dopamine deficiency to study compensatory neurodegenerative mechanisms. J Neurosci Methods 308: 205-218, 2018.

IMBRIANI P, SCIAMANNA G, SANTORO M, SCHIRINZI T, PISANI A: Promising rodent models in Parkinson's disease. Parkinsonism Relat Disord 46: 10-14, 2018. 
JAKARIA M, PARK SY, HAQUE ME, KARTHIVASHAN G, KIM IS, GANESAN P, CHOI DK: Neurotoxic AgentInduced Injury in Neurodegenerative Disease Model: Focus on Involvement of Glutamate Receptors. Front Mol Neurosci 11: 307, 2018.

JIANG P \& DICKSON DW: Parkinson's disease: experimental models and reality. Acta Neuropathol 135: 13-32, 2018.

JOHNSON M E, LIM Y, SENTHILKUMARAN M, ZHOU XF, BOBROVSKAYA L: Investigation of tyrosine hydroxylase and BDNF in a low-dose rotenone model of Parkinson's disease. J Chem Neuroanat 70: 33-41, 2015.

KIM HD, JEONG KH, JUNG UJ, KIM SR: Myricitrin ameliorates 6-hydroxy- dopamine-induced dopaminergic neuronal loss in the substantia nigra of mouse brain. J Med Food 19: 374-382, 2016.

MARIN C, BONASTRE M, MENGOD G, CORTES R, GIRALT A, OBESO JA, SCHAPIRA AH: Early L-dopa, but not pramipexole, restores basal ganglia activity in partially 6-OHDA-lesioned rats. Neurobiol Dis 64: 36-47, 2014.

MARIN C, BONASTRE M, MENGOD G, CORTES R, RODRIGUEZ-OROZ MC: From unilateral to bilateral parkinsonism: Effects of lateralization on dyskinesias and associated molecular mechanisms. Neuropharmacology 97: 365-375, 2015.

FORNAI M, PELLEGRINI C, ANTONIOLI L, SEGNANI C, IPPOLITO C, BAROCELLI E, BALLABENI V, VEGEZZI G, AL HARRAQ Z, BLANDINI F, LEVANDIS G, CERRI S, BLANDIZZI C, BERNARDINI N, COLUCCI R: Enteric dysfunctions in experimental Parkinson's disease: alterations of excitatory cholinergic neurotransmission regulating colonic motility in rats. J Pharmacol Exp Ther 356: 434-444, 2016.

PELLEGRINI C, FORNAI M, COLUCCI R, TIROTTA E, BLANDINI F, LEVANDIS G, CERRI S, SEGNANI C, IPPOLITO C, BERNARDINI N, CSERI K, BLANDIZZI C, HASKÓ G, ANTONIOLI L: Alteration of colonic excitatory tachykininergic motility and enteric inflammation following dopaminergic nigrostriatal neurodegeneration. J Neuroinflam 13: 146, 2016.

MOUSSAOUI N, LARAUCHE M, BIRAUD M, MOLET J, MILLION M, MAYER E, TACHE Y: Limited Nesting Stress Alters Maternal Behavior and In Vivo Intestinal Permeability in Male Wistar Pup Rats. PLoS One 11: $0155037,2016$.

NATALE G, KASTSIUSHENKA O, FULCERI F, RUGGIERI S, PAPARELLI A, FORNAI F: MPTP-induced parkinsonism extends to a subclass of TH-positive neurons in the gut. Brain Res 1355: 195-206, 2010.

NETUSHA T, ALICJA P, CHRISTIAN S, AVEE N, SZAMOSI JC, CHRIS PV, DESSI L, LOUIS PS, JENNIFER J, KEVIN PF, JONATHAN DS, MAGGIE JL, DONALD JD, ELENA FV, MICHAEL GS, DAWN MEB: Age-Associated Microbial Dysbiosis Promotes Intestinal Permeability, Systemic Inflammation, and Macrophage Dysfunction. Cell Host Microbe 21: 455-466, 2017.

ODIN P, CHAUDHURI KR, VOLKMANN J, ANTONINI A, STORCH A, DIETRICHS E, PIROSEK Z, HENRIKSEN T, HORNE M, DEVOS D, BERGQUIST F: Viewpoint and practical recommendations from a movement disorder specialist panel on objective measurement in the clinical management of Parkinson's disease. NPJ Parkinsons Dis 4: 14, 2018.

SAUERBIER A, JITKRITSADAKUL O, TITOVA N, KLINGELHOEFER L, TSUBOI Y, CARR H, KUMAR H, BANERJEE R, ERRO R, BHIDAYASIRI R, SCHRAG A, ZIS P, LIM SY, AL-HASHEL JY, KAMEL WA, MARTINEZ MARTIN P, RAYY CHAUDHURI K: Non-Motor Symptoms Assessed by Non-Motor Symptoms Questionnaire and Non-Motor Symptoms Scale in Parkinson's Disease in Selected Asian Populations. Neuroepidemiology 49: 1-17, 2017.

SCHNEIDER JS, PIOLI EY, JIANZHONG Y, LI Q, BEZARD E: Levodopa improves motor deficits but can further disrupt cognition in a macaque Parkinson model. Mov Disord 28: 663-667, 2013.

SHEN DF, TIAN XY, ZHANG BB, SONG RR: Mechanistic evaluation of neuroprotective effect of estradiol on rotenone and 6-OHDA induced Parkinson's disease. Pharmacol Rep 69: 1178-1185, 2017.

SHIREEN E, PERVEZ S, MASROOR M, ALI WB, RAIS Q, KHALIL S, TARIQ A, HALEEM DJ: Reversal of haloperidol induced motor deficits in rats exposed to repeated immobilization stress. Pak J Pharm Sci 27 : 1459-1466, 2014.

STEWARD MC, ISHIGURO H, CASE RM: Mechanisms of bicarbonate secretion in the pancreatic duct. Annu Rev Physiol 67: 377-409, 2005. 
SUN M, HE C, CONG Y, LIU Z: Regulatory immune cells in regulation of intestinal inflammatory response to microbiota. Mucosal Immunol 8: 969-978, 2015.

TIAN YM, CHEN X, LUO DZ, ZHANG XH, XUE H, ZHENG LF, YANG N, WANG, XM, ZHU JX: Alteration of dopaminergic markers in gastrointestinal tract of different rodent models of Parkinson's disease. Neuroscience 153: 634-644, 2008.

TULSTRUP MV, CHRISTENSEN EG, CARVALHO V, LINNINGE C, AHRNÉ S, HØJBERG O, LICHT TR, BAHL MI: Antibiotic treatment affects intestinal permeability and gut microbial composition in Wistar rats dependent on antibiotic class. PLOS one 10: 0144854, 2015.

ZHANG XL, LI Y, LIU CZ, FAN RF, WANG P, ZHENG LF, HONG F, FENG XY, ZHANG Y, LI LS, ZHU JX: Alteration of enteric monoamines with monoamine receptors and colonic dysmotility in 6-hydroxydopamine induced Parkinson's disease rats. Transl Res 66: 152-162, 2015.

ZHENG LF, WANG ZY, LI XF, SONG J, HONG F, LIAN H, WANG Q, FENG XY, TANG YY, ZHANG Y, ZHU JX: Reduced expression of choline acetyltransferase in vagal motoneurons and gastric motor dysfunction in a 6-OHDA rat model of Parkinson's disease. Brain Res 1420: 59-67, 2011.

ZHENG LF, SONG J, FAN RF, CHEN CL, REN QZ, ZHANG X L, FENG XY, ZHANG Y, LI LS, ZHU JX: The role of the vagal pathway and gastric dopamine in the gastroparesis of rats after a 6-hydroxydopamine microinjection in the substantia nigra. Acta Physiol 211: 434-446, 2014.

ZHENG Z, TRAVAGLI RA: Dopamine effects on identified rat vagal motoneurons. Am J Physiol Gastrointest Liver Physiol 292, 1002-1008, 2007.

ZIS P, ERRO R, WALTON CC, SAUERBIER A, CHAUDHURI KR: The range and nature of non-motor symptoms in drug-naive Parkinson's disease patients: a state-of-the-art systematic review. NPJ Parkinsons Dis 1: 15013, 2015 . 